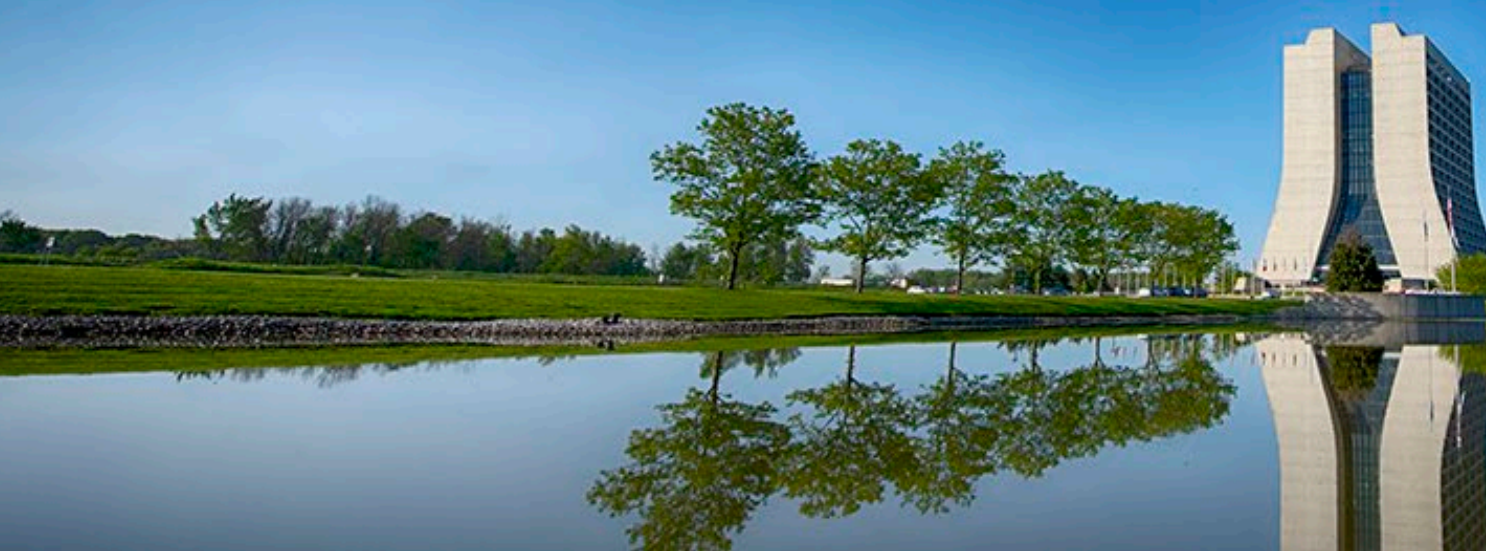

\title{
High Power Targets
}

Challenges for next-generation high-intensity neutrino beams

Kavin Ammigan

The $22^{\text {nd }}$ International Workshop on Neutrinos from Accelerators (NuFact 2021) 10 September 2021 


\section{Robust High-Power Targets Critical in Maximizing the Efficiency of Neutrino Production}

Recently, major accelerator facilities have been limited in beam power not by their accelerators, but by target survivability concerns

- NuMI-MINOS, FNAL (2010-11)

- $\quad$ Reduced beam power (-10\% to $-40 \%)$

- Target failures attributed to faulty welds

- MLF, J-PARC (2015-16)

- $\quad$ Early replacement of target

- $\quad$ Limited to $200 \mathrm{~kW}$ when resuming ops

- SNS, ORNL

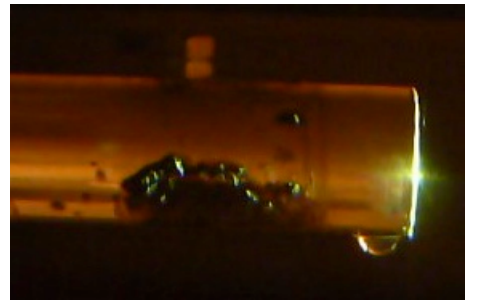

MINOS NT-01 target (FNAL)

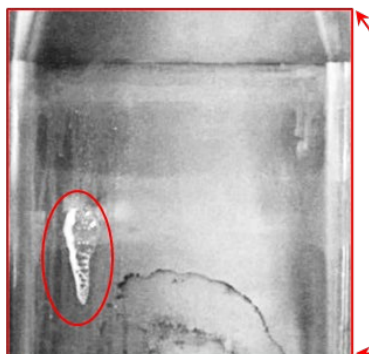

- $\quad$ Reduced beam power (-15\%) frequently in 2013-14

- Target vessel failures attributed to faulty welds and dynamic stresses

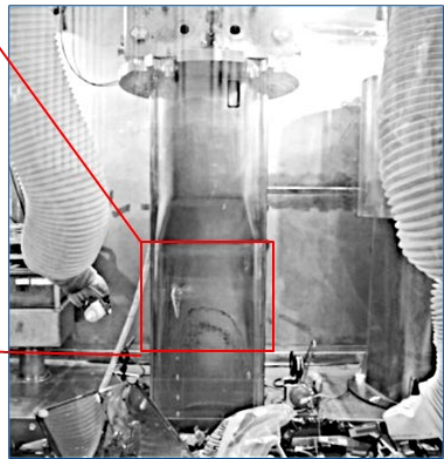
SNS target vessel (ORNL)

Next-generation multi-MW accelerator target facilities present even greater challenges LBNF DUNE 1.2-2.4 MW, Hyper-K 1.3 MW, Future neutrino facilities 4 MW+

Target R\&D essential to:

- Avoid compromising particle production efficiency by limiting beam parameters

- Maintain reliable operation and accurately predict component lifetime 


\section{What we want to avoid...}
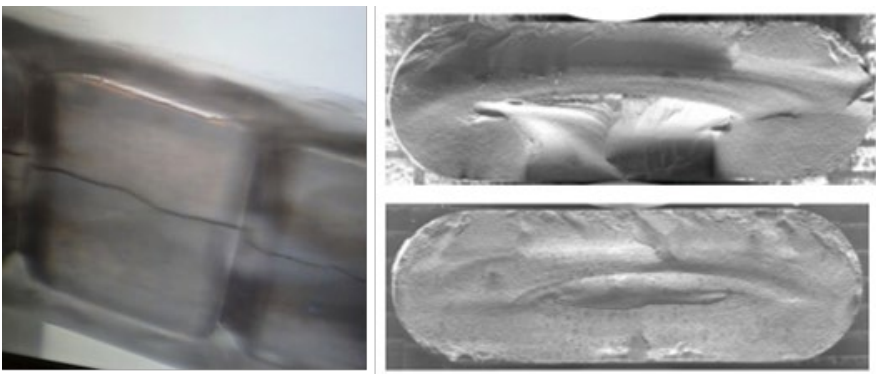

MINOS NT-02 target failure: radiation-induced swelling (FNAL)

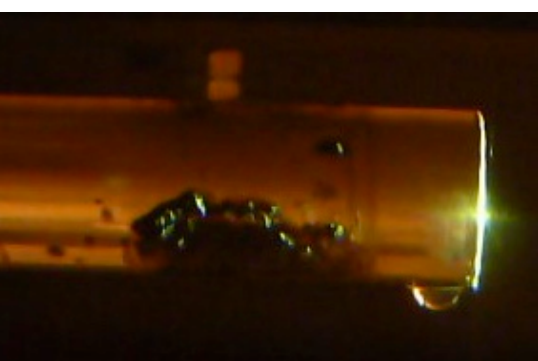

MINOS NT-01 target containment water leak (FNAL)

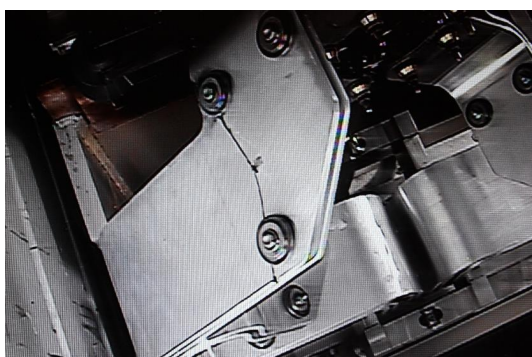

Horn stripline fatigue failure (FNAL)

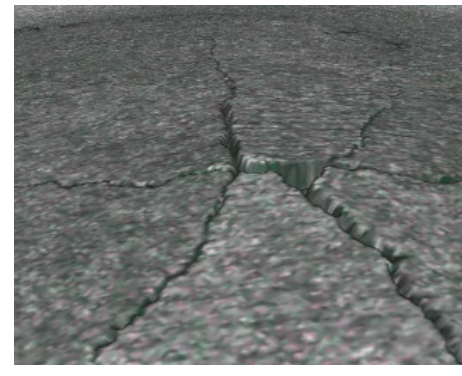

Be window embrittlement (FNAL)

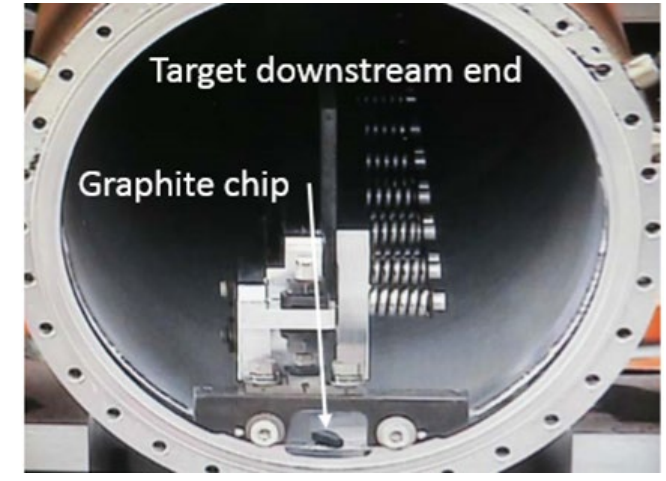

NOvA MET-01 target fin fracture (FNAL)

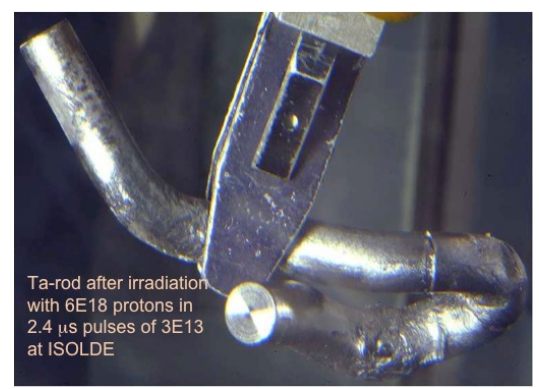

ISOLDE target (CERN)

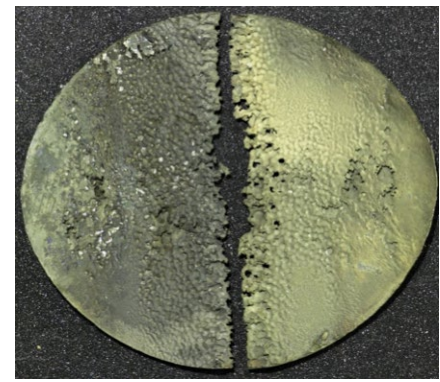

Target containment vessel cavitation (ORNL - SNS) 


\section{High Power Targetry Scope}
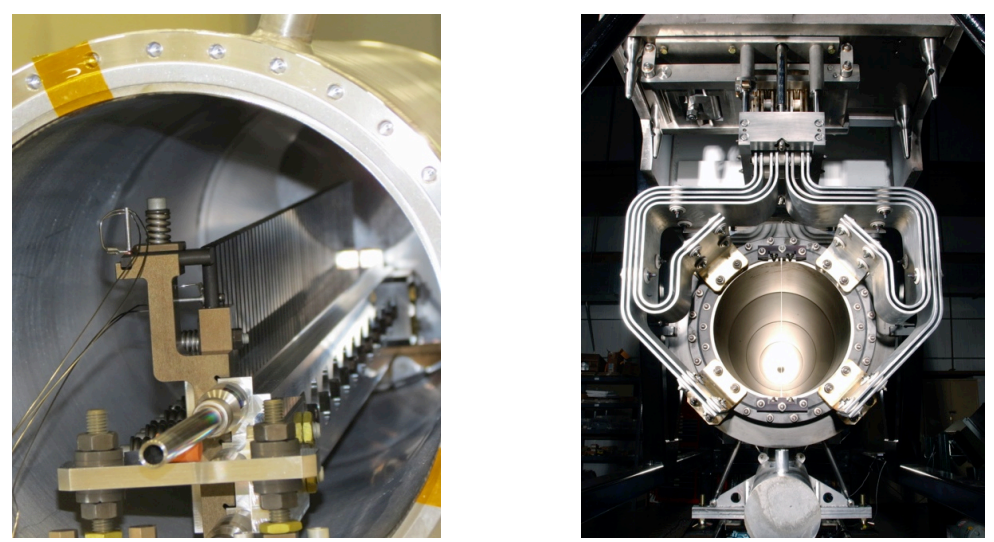

- Target

- Solid, Liquid, Fixed, Rotating

- Facility Requirements

- Remote Handling

- Shielding \& Radiation Transport

- Air Handling

- Cooling System
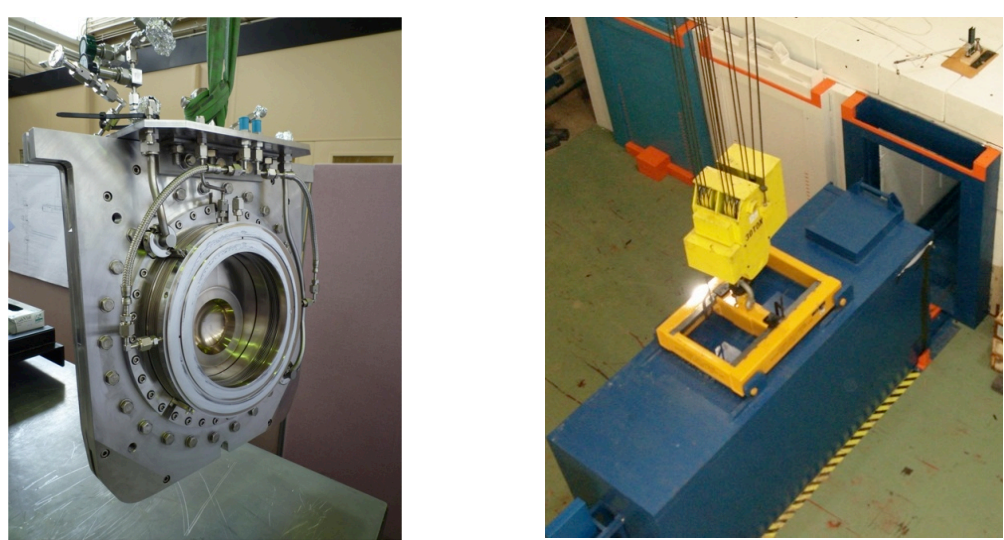

- Other beam-intercepting devices

- Collimators

- Collection optics (horns, solenoids)

- Monitors \& Instrumentation

- Beam windows

- Absorbers 


\section{High Power/Intensity Targetry Challenges}
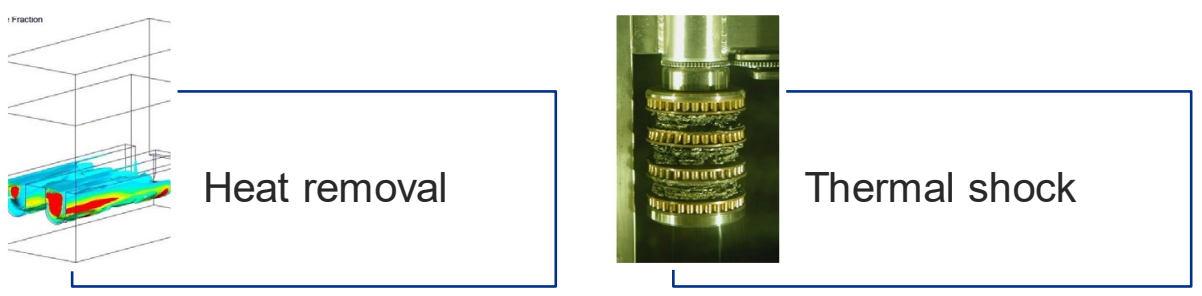

Thermal Shock and Radiation Damage identified as most cross-cutting challenges of high-power target facilities
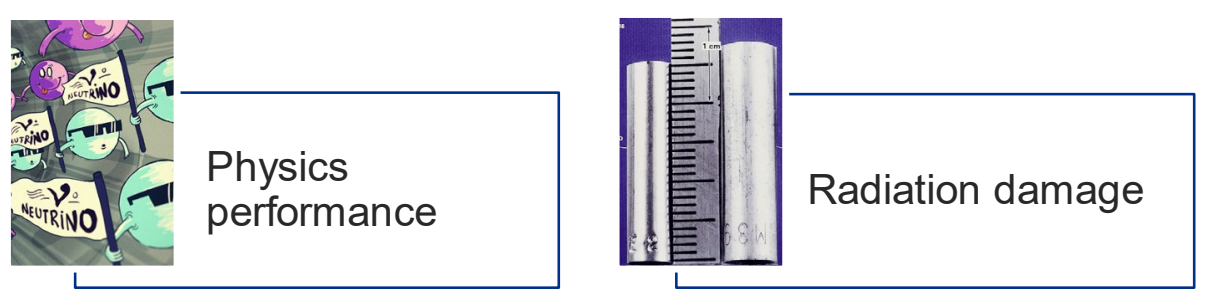

Additional neutrino beams challenges

- Primary beam handling and instrumentation

- Accuracy and consistency of beam inputs
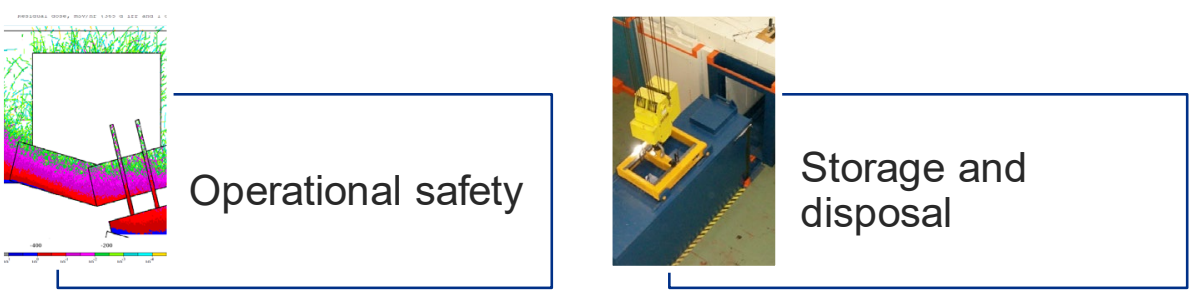

- Focusing elements

- Beam-based alignment

- Secondary beam instrumentation

- Hadron production 


\section{Radiation Damage in Materials}

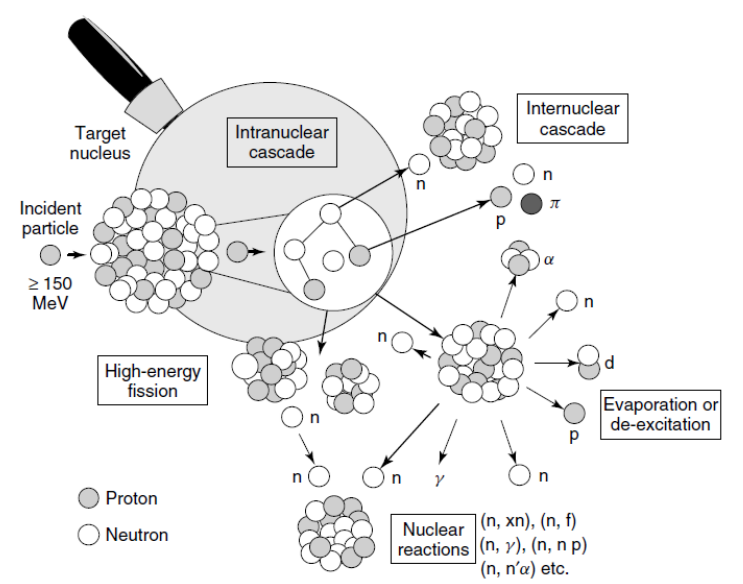

From D. Filges, F. Goldenbaum, in:, Handb. Spallation Res Wiley-VCH Verlag GmbH \& Co. KGaA, 2010, pp. 1-61.

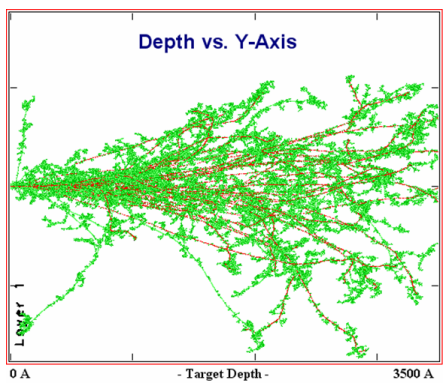

Beam-induced damage to the microstructure

- Atomic displacements (cascades)

- Displacement Per Atom (DPA) = Average number of stable interstitial/vacancy pairs created

- Creation and agglomeration of point defects

- Segregation (precipitation) or depletion of point defect sinks

- Creation of transmutation products ( $\mathrm{H}$ and He production)
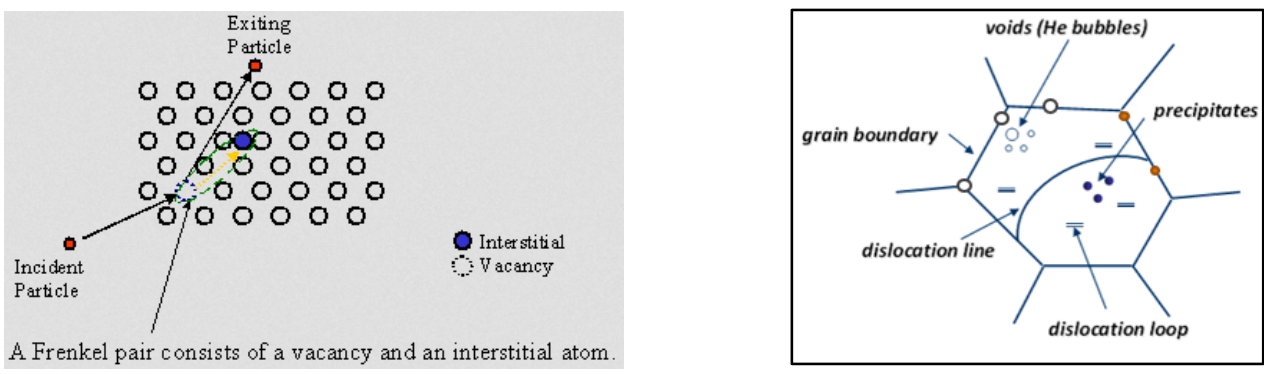

草 Fermilab 


\section{Radiation Damage Effects}

- Atomic displacements in crystal lattice (DPA) cause bulk property changes

- Embrittlement

- Creep

- Swelling

- Fracture toughness reduction

- Thermal/electrical conductivity reduction

- Coefficient of thermal expansion

- Modulus of Elasticity

- Accelerated corrosion

- Transmutation products

- $\mathrm{H}$, He gas production causes void formation and embrittlement

- Radiation damage effects very dependent upon material and irradiation conditions

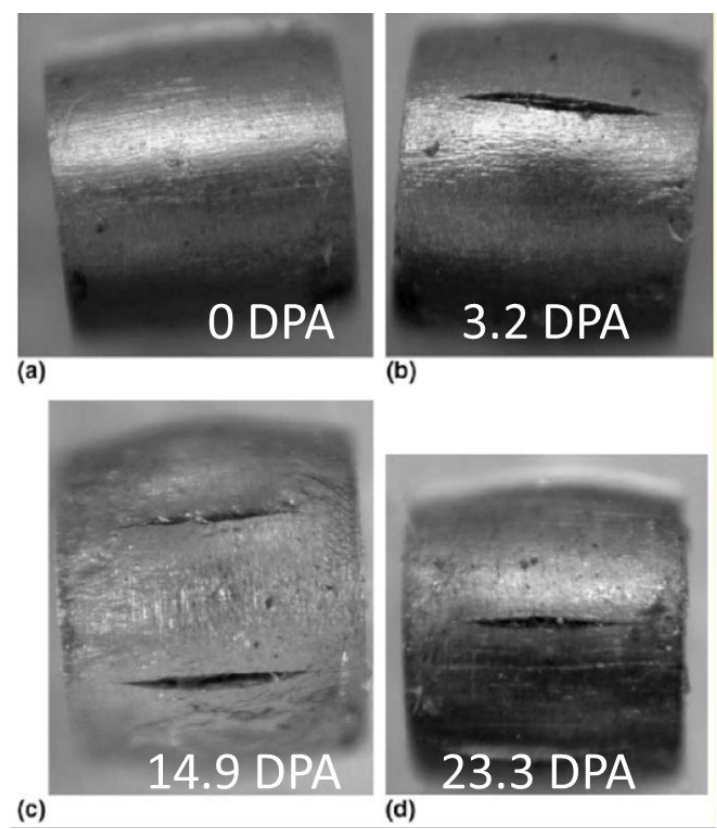

S.A. Maloy et al., J. Nuclear Materials, 343, pp. 219-226, 2005

- Temperature, dose rate, particle energy/type 


\section{Radiation Damage Effects}

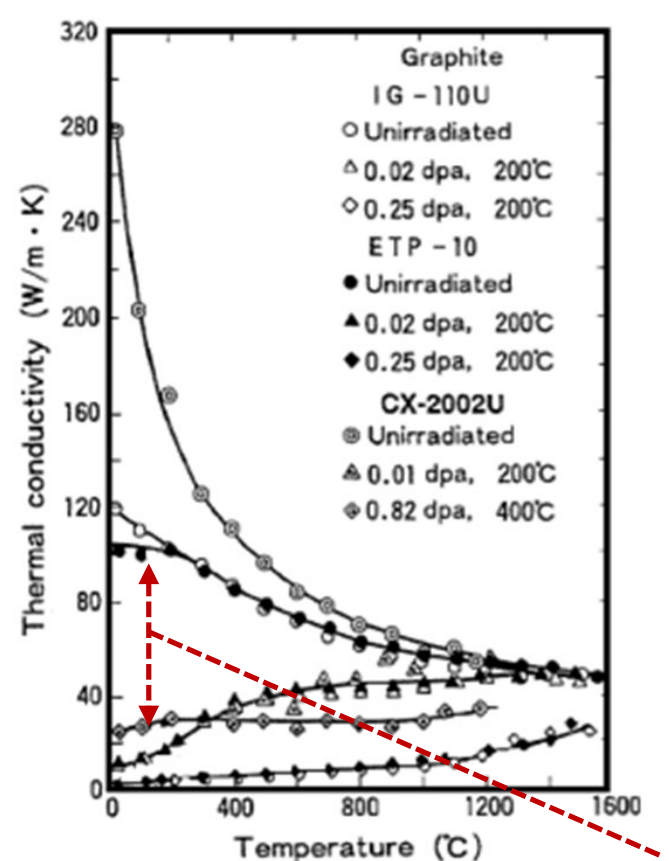

N. Maruyama and M. Harayama, Journal of Nuclear Materials, 195, 44-50 (1992)

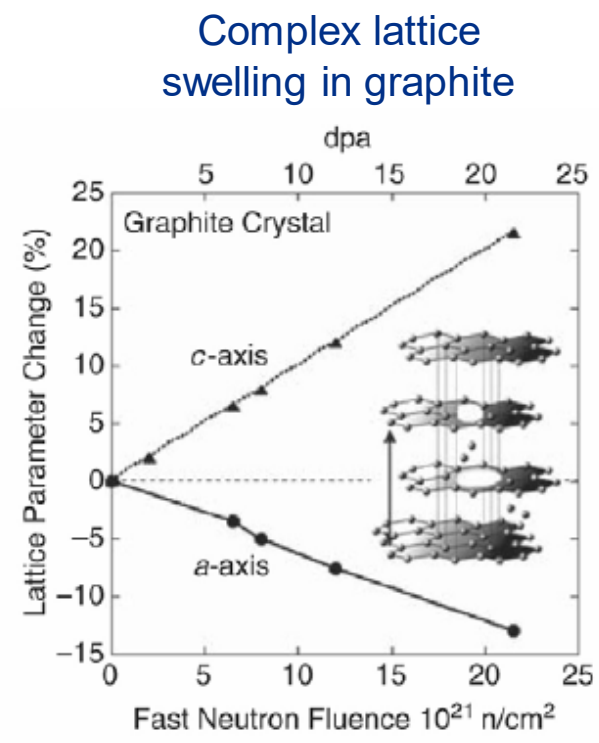

Factor of 10 reduction in thermal conductivity of graphite after 0.02 DPA

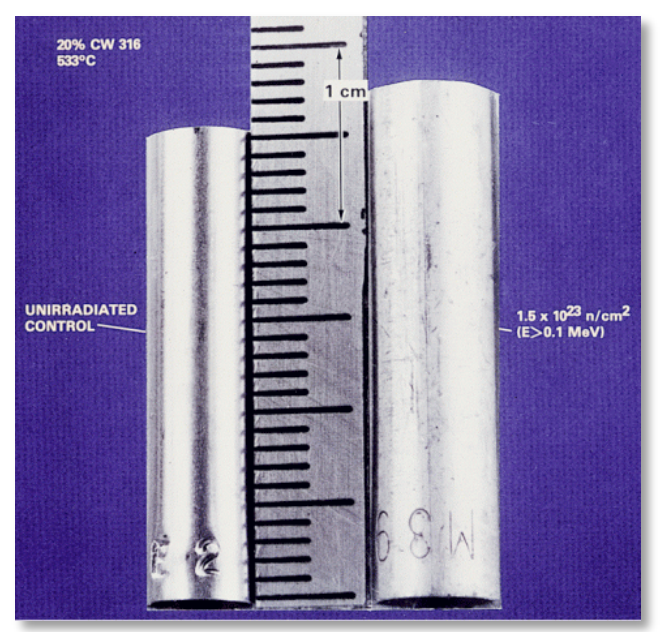

D.L. Porter and F. A. Garner, J. Nuclear Materials, 159, p. 114 (1988)

Void swelling in 316 Stainless Steel tube exposed to reactor dose of $1.5 \times 10^{23} \mathrm{n} / \mathrm{cm}^{2}$ 


\section{Thermal Shock Effects (stress waves)}

- High-intensity pulsed beam creates localized area of compressive stress, generated due to fast expansion of material surrounded by cooler material

- $\quad$ NuMl 1-MW graphite target: $\Delta \mathrm{T} \sim 250 \mathrm{~K}$ in $10 \mu \mathrm{s}\left(2.5 \times 10^{7} \mathrm{~K} / \mathrm{s}\right)$

- Dynamic stress waves travel through the target at sonic velocities

- Thermal shock can induce plastic deformation, cracking and fatigue failure

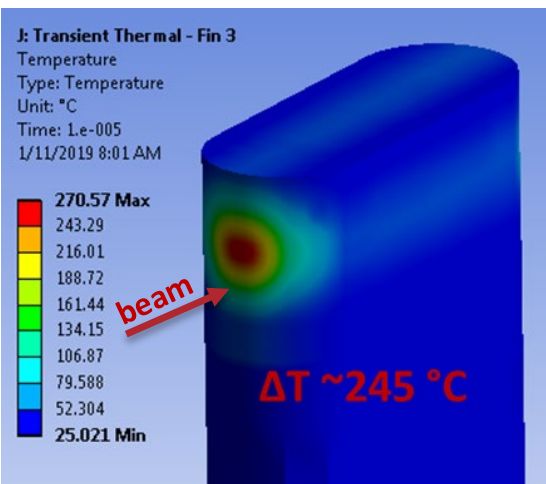

Temperature rise during $10 \mu$ s pulse in NuMl 1-MW graphite target (FNAL)
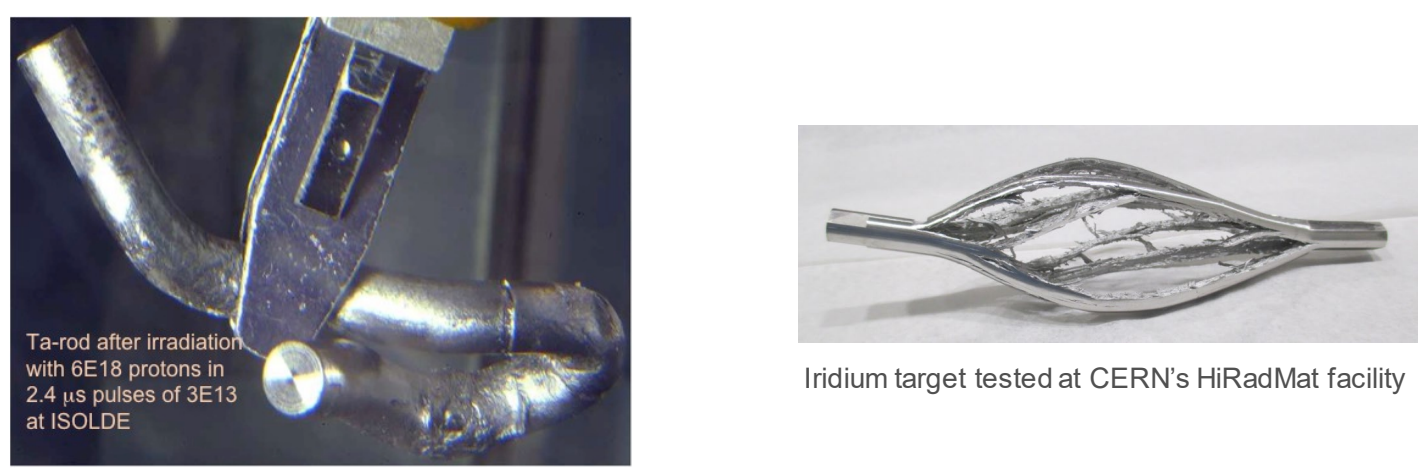

Iridium target tested at CERN's HiRadMat facility

Ta-rod after irradiation with $6 \times 10^{18}$ protons in $2.4 \mu \mathrm{s}$ pulses of $3 \times 10^{13}$ at ISOLDE (photo: J. Lettry) 


\section{Stress Wave Example: T2K Window}

T2K Titanium beam window

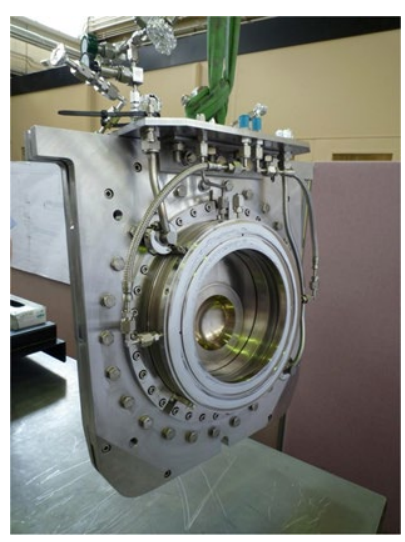

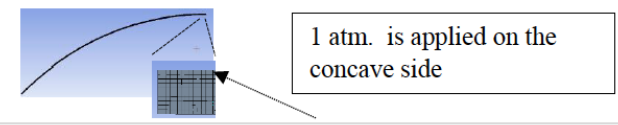

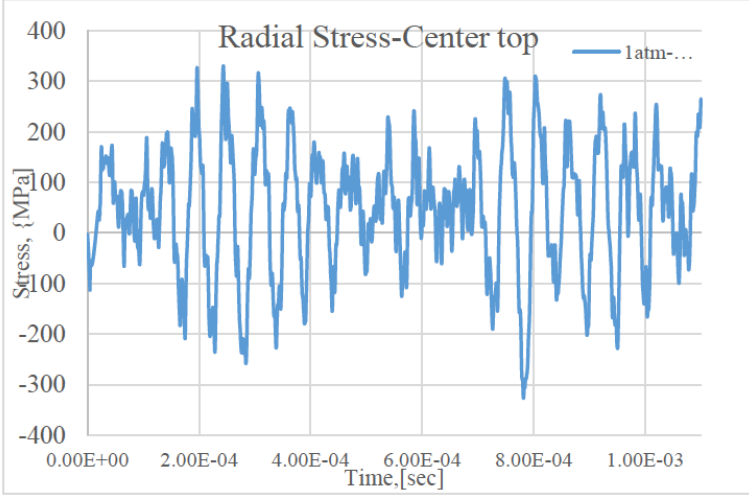

Material response depends on:

$>$ Specific heat (temperature jump)

Coefficient of thermal expansion (strain)

$>$ Modulus of elasticity (stress)

Flow stress behavior (plastic deformation)

$>$ Strength limits (yield, fatigue, fracture toughness)

$\sigma=\sqrt{\rho E} \cdot \alpha \cdot L \cdot \frac{\Delta T}{\Delta t} \quad \begin{aligned} & \text { Initial stress } \\ & \text { wave amplitude }\end{aligned}$

- Cyclic stress loading environment can lead to fatigue failure

- Heavy dependence on material properties

$$
c=\sqrt{\frac{E}{\rho}} \quad \begin{array}{ll}
\text { Elastic wave } \\
\text { speed }
\end{array}
$$




\section{Radiation Damage Data}

- Use of data from nuclear materials research is limited and cannot be directly utilized

- Effects from low-energy neutron irradiations do not equal effects from high-energy proton irradiations

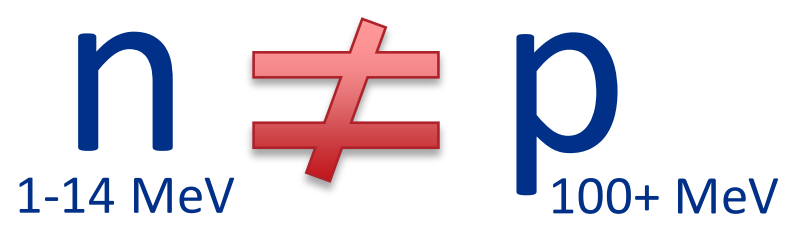

Irradiation DPA rate (DPA/s) He gas production Irradiation Temp Source (appm/DPA)

$\left({ }^{\circ} \mathrm{C}\right)$

Mixed spectrum fission reactor

$3 \times 10^{-7}$

$1 \times 10^{-1}$

200-600

Fusion reactor

$1 \times 10^{-6}$

$1 \times 10^{1}$

400-1000

High energy proton beam 


\section{Neutrino HPT R\&D Materials Exploratory Map}

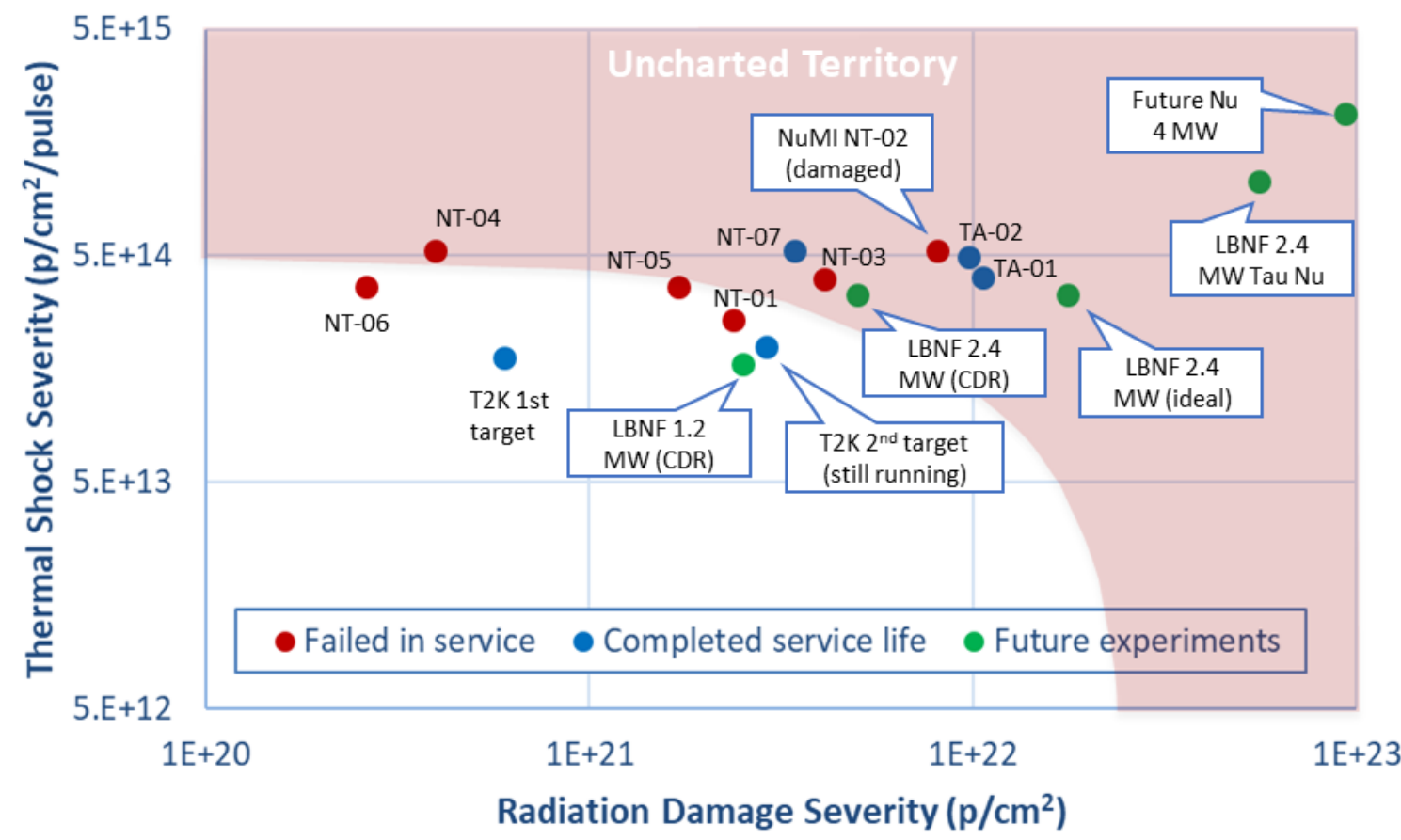

$\sim 10 x$ increase in accumulated proton fluence expected in future multi-MW facilities 


\section{$\because \quad R$ a D I A T E Collaboration}

\section{diation Damage In Accelerator arget Environments}

Objective:

- Harness existing expertise in nuclear materials and accelerator targets

- Generate new and useful materials data for application within the accelerator and fission/fusion communities

Activities include:

- Analysis of materials taken from existing beamline as well as new irradiations of candidate target materials at low and high energy beam facilities

- In-beam thermal shock experiments

Program manager: Dr. Frederique Pellemoine (FNAL)

\section{Fermilab}

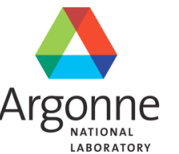

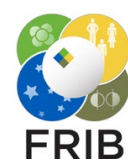

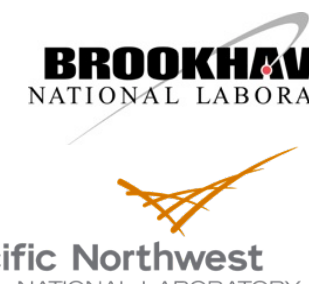

OAK

RIDGE

\section{radiate.fnal.gov}

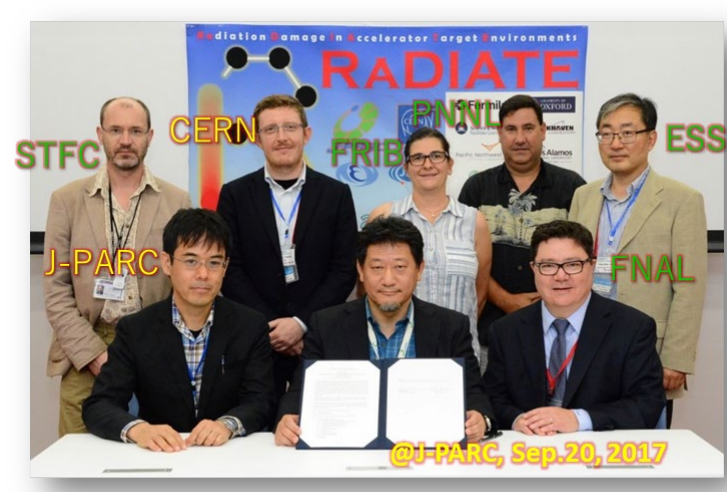

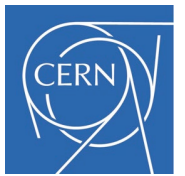

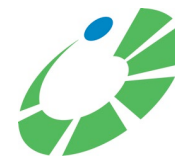

Crement 


\section{High Power Target Materials R\&D}

Examine targets and beam window materials behavior under prototypic multi-MW proton beam conditions

- Graphite (target core) studies:

- Beam-induced swelling and fracture studies

- High-dose ion irradiation of graphite

- Beryllium (beam window) studies:

- NuMI beam window analysis \& Helium ion implantation

- Post-irradiation examination of BLIP-irradiated specimens

- In-beam thermal shock testing at CERN's HiRadMat facility

Benefits to multi-MW targets

- Alloy/grade and heat treatment choice

- Identify novel candidate materials

- Cooling system design and operating temperature

- Tolerable beam intensities

- Expected lifetimes

- Titanium (beam window) studies:

- Tensile testing of BLIP-irradiated specimens

- Low-energy ion irradiation and nano-indentation

- World first high-cycle fatigue testing of irradiated titanium at FNAL

- Novel materials studies:

- Electro-spun nanofibers, high-entropy alloys, metal foams, MoGr, highly-ductile TFGR tungsten 


\section{Analysis of Fractured NuMI Target Fin}

NuMl target (NT-02) autopsy and examination

- Peak fluence: $8 \times 10^{21} \mathrm{p} / \mathrm{cm}^{2}$

- Beam energy: $120 \mathrm{GeV}$

- Spill duration: $10 \mu \mathrm{s}, 4 \times 10^{13}$ protons/pulse

- Duty cycle: $1.87 \mathrm{~s}$

- Estimated peak DPA: 0.63
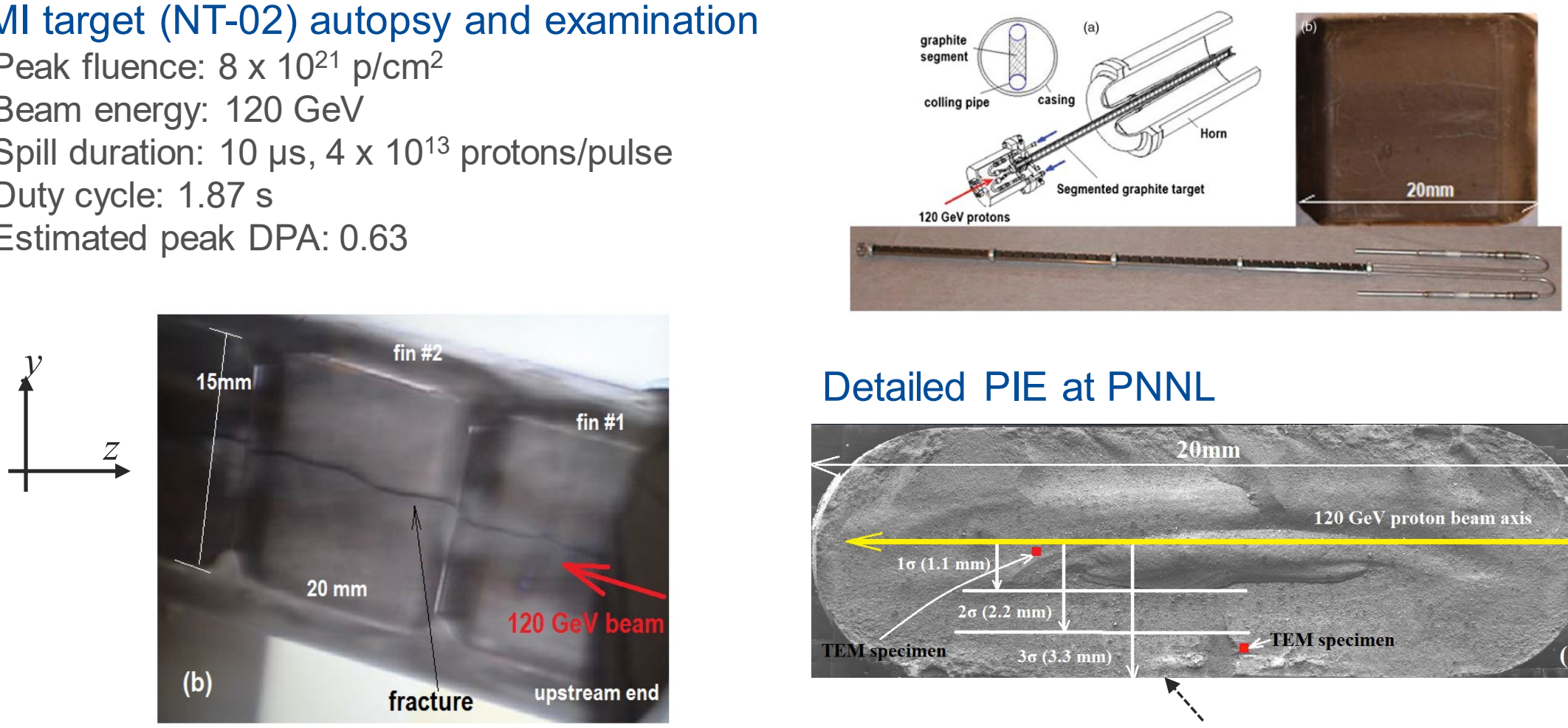

Detailed PIE at PNNL

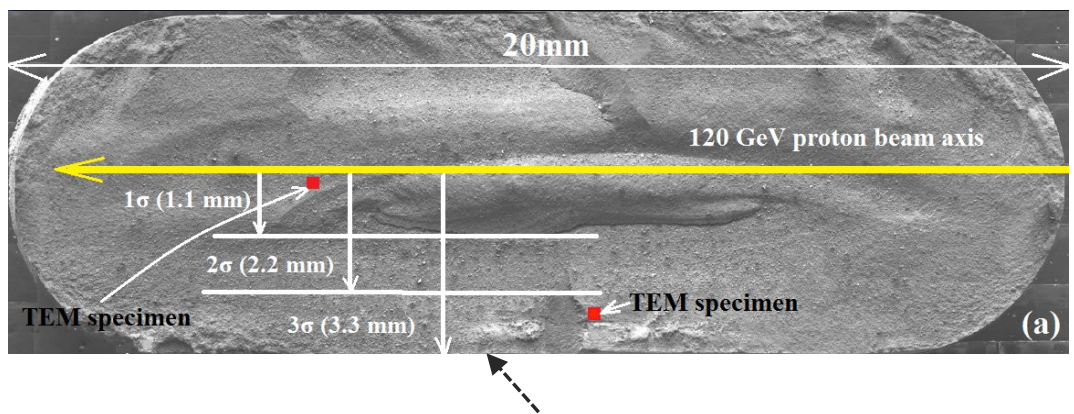

Bulk swelling of $\sim 4 \%$ 


\section{X-Ray Diffraction of NuMI Graphite Fin at NSLS-II}
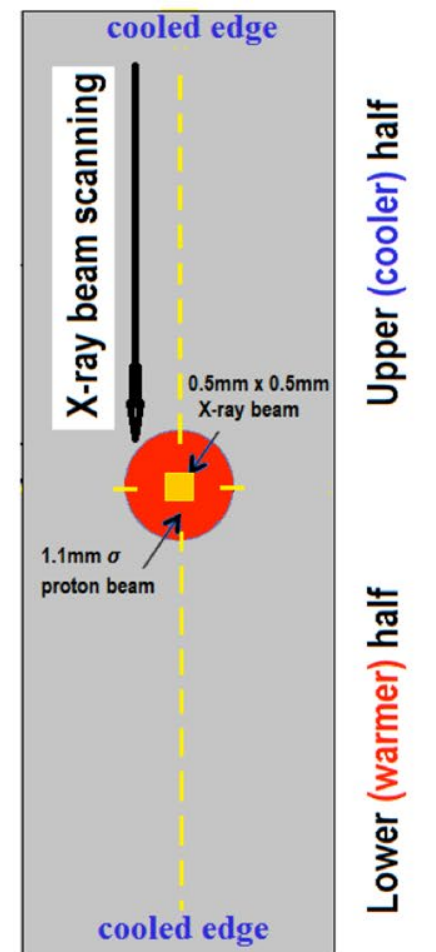

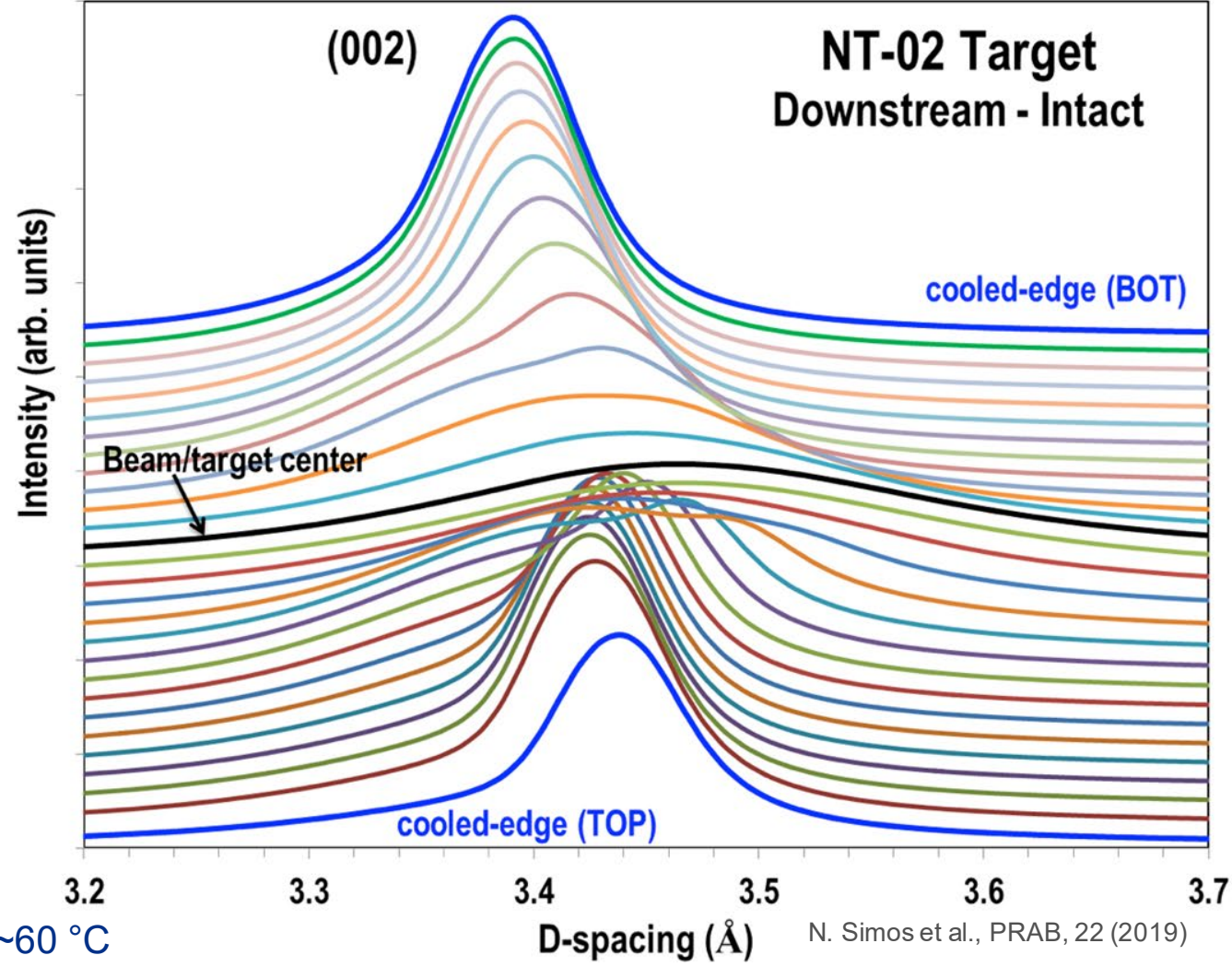

XRD shows lattice growth and amorphization at the beam center

Irradiation temperature $\sim 60{ }^{\circ} \mathrm{C}$ (330 ${ }^{\circ} \mathrm{C}$ during pulse) 


\section{NuMI Beryllium Window Analysis}
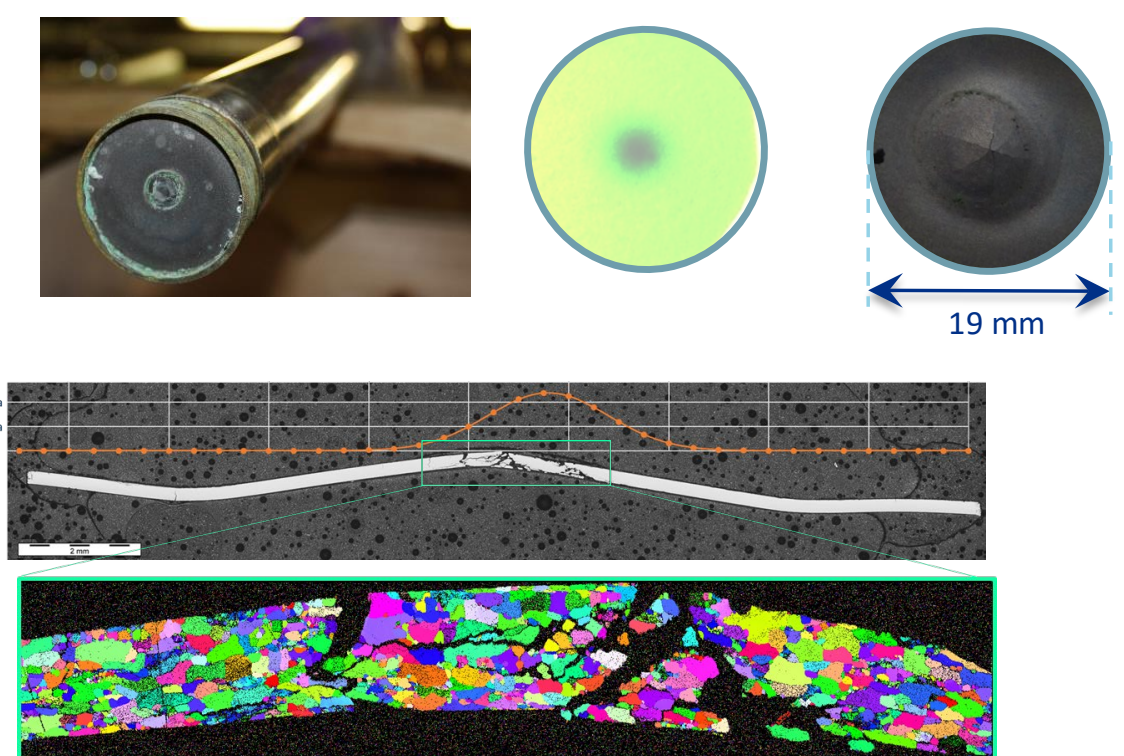

- Observed transition from transgranular fracture to grain boundary/mixed mode fracture in irradiated $\mathrm{Be}$

V.Kuksenko et al. J. Nuclear Materials, 490, pp.260-271 (2017)

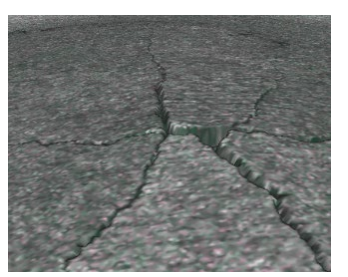

- $120 \mathrm{GeV}$ proton beam

- 1.54 x $10^{21}$ POT (0.5 peak DPA)

- $\mathrm{T} \sim 50{ }^{\circ} \mathrm{C}$

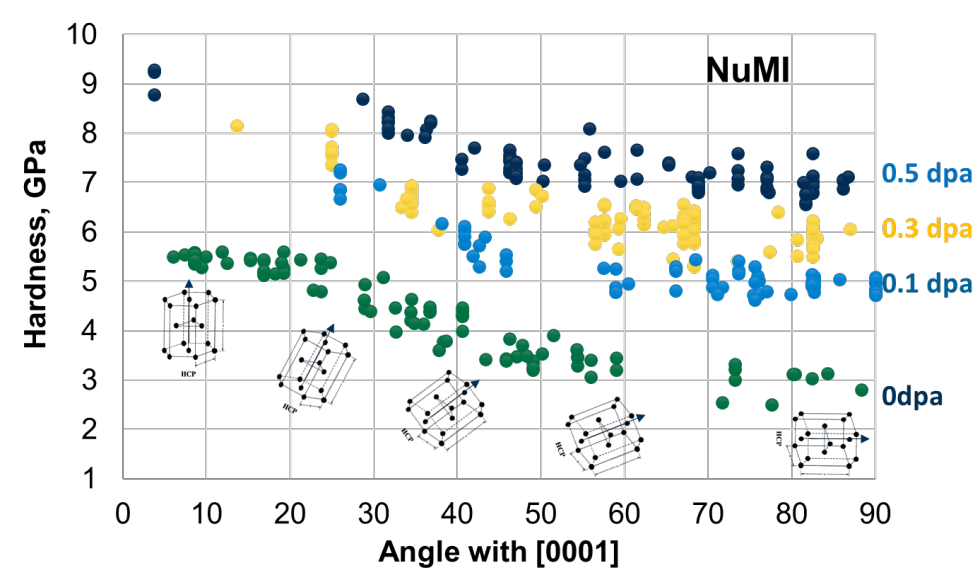

- Significant hardening even at 0.1 DPA

- Hardness of irradiated Be less anisotropic

- Increased hardness means less ductility (more brittle) 


\section{High Energy Proton Irradiation at BNL's BLIP Facility}

- Unique facility for material irradiation in tandem with medical isotope production

- High energy protons: 66 - $200 \mathrm{MeV}$ with $165 \mu \mathrm{A}$ peak current

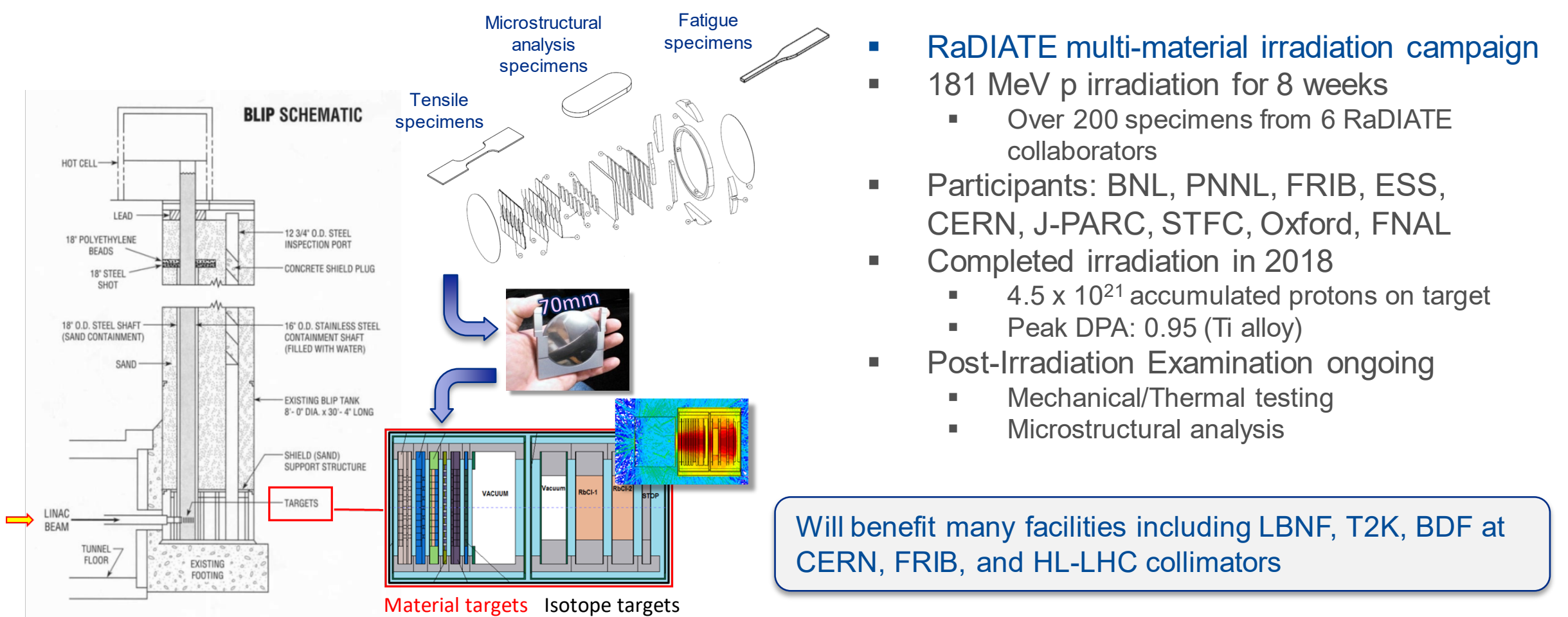




\section{BLIP Ti Alloy Tensile Testing}

Stress-strain curves for Ti-6Al-4V (left) and Ti-3Al-2.5V (right)
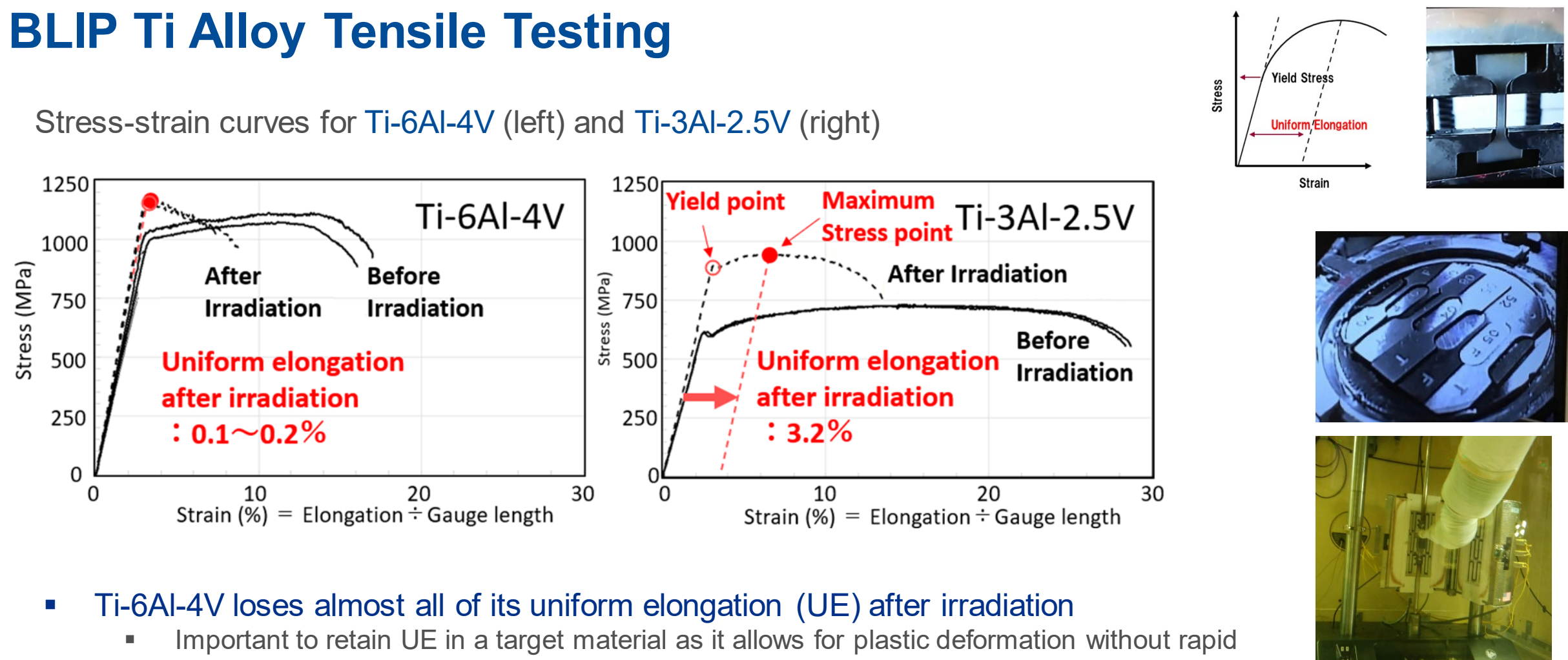

- Ti-6Al-4V loses almost all of its uniform elongation (UE) after irradiation

- Important to retain UE in a target material as it allows for plastic deformation without rapid growth of cracks and sudden failure

Testing done in hot cell

- Evidence that Ti-3AI-2.5V alloy is more radiation-tolerant 


\section{High-Cycle Fatigue Testing of Irradiated Ti Alloys}

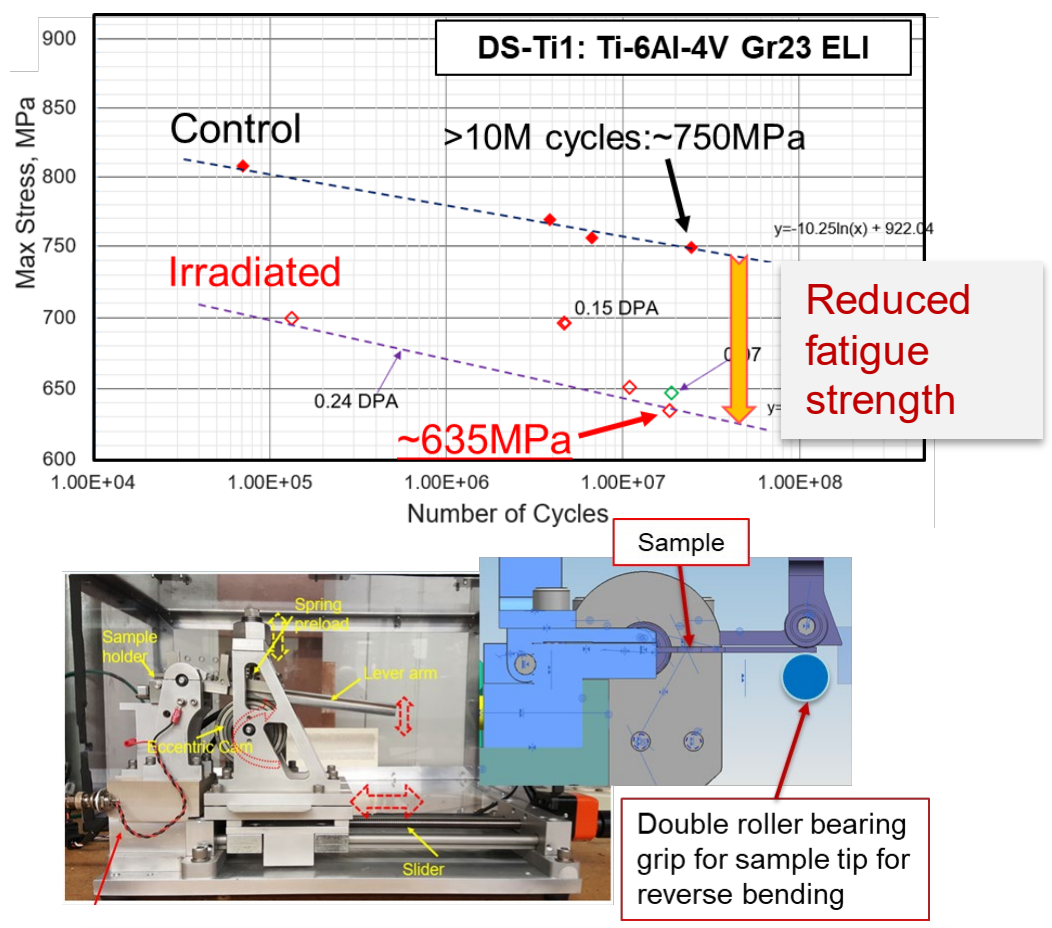

$3^{\text {rd }}$ Generation Fatigue Testing Machine (FTM) under development
Ultrasonic mesoscale Fatigue Rig (UFR) at the UKAEA-MRF

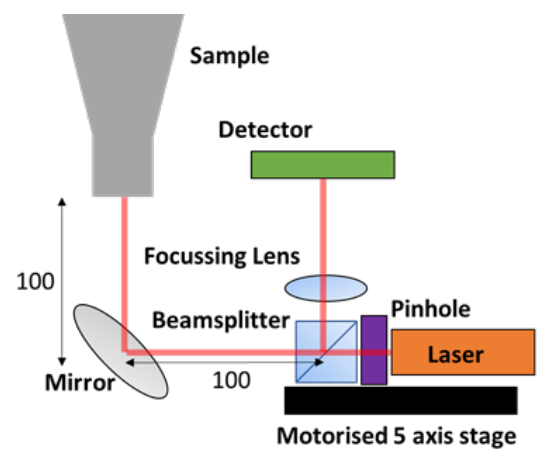

Extraction of meso-fatigue foil from BLIP capsule in PNNL hot cell

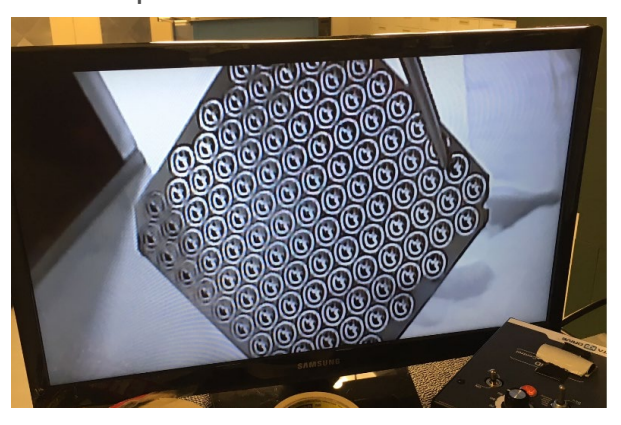

$20 \mathrm{kHz}=10^{8}$ cycles in $1.5 \mathrm{~h}$
BLIP 2017-2018 Ti foils have been shipped from PNNL to MRF for testing over the coming months 


\section{Low-Energy lon Irradiation}

Alternative method to mimic high-energy proton-induced radiation damage

- High damage (DPA) accumulation in short time (without activation)

- Shallow damage depth (use of micro-mechanics and meso-scale testing)

- Dual/Triple beams irradiation needed to reproduce transmutation gas production

Graphite irradiation with $4.5 \mathrm{MeV} \mathrm{He}^{++}$ions

A. Burleigh and Prof. J. Terry (IIT)

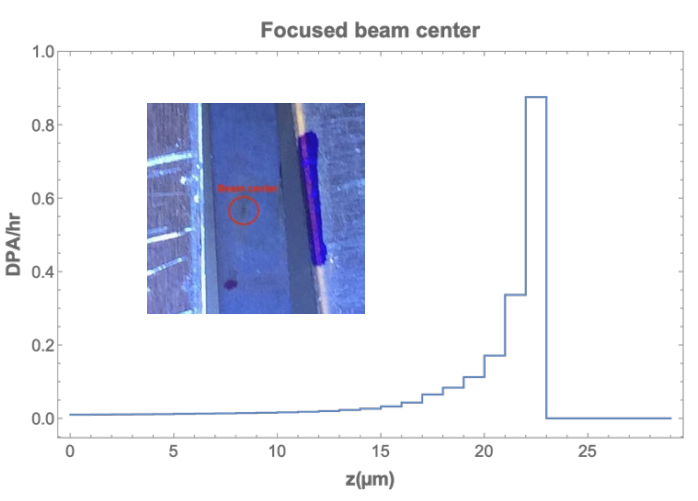

AFM measurements show bulk swelling of $\sim 3.8 \mu \mathrm{m}$ in the irradiated region
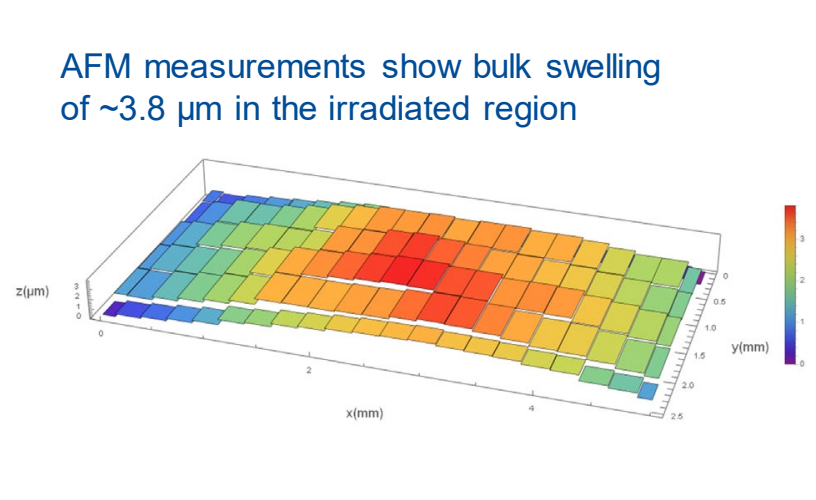

TEM: e- diffraction shows increase in d-spacing and decrease in a-spacing, similar to failed NT-02 target

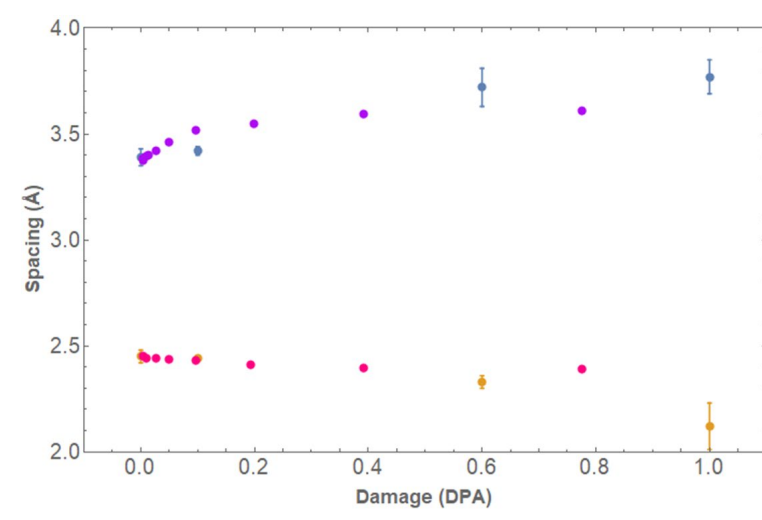

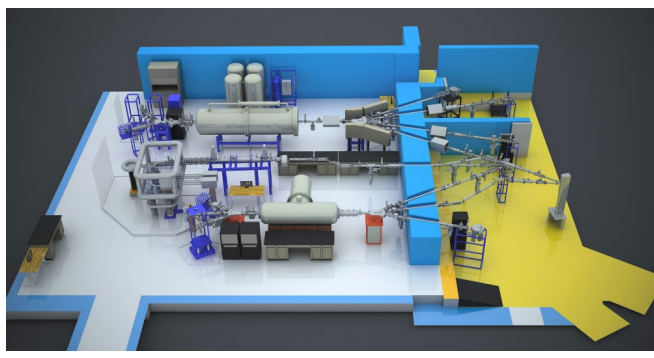

Michigan Ion Beam Laboratory 


\section{Helium Implantation in Beryllium}
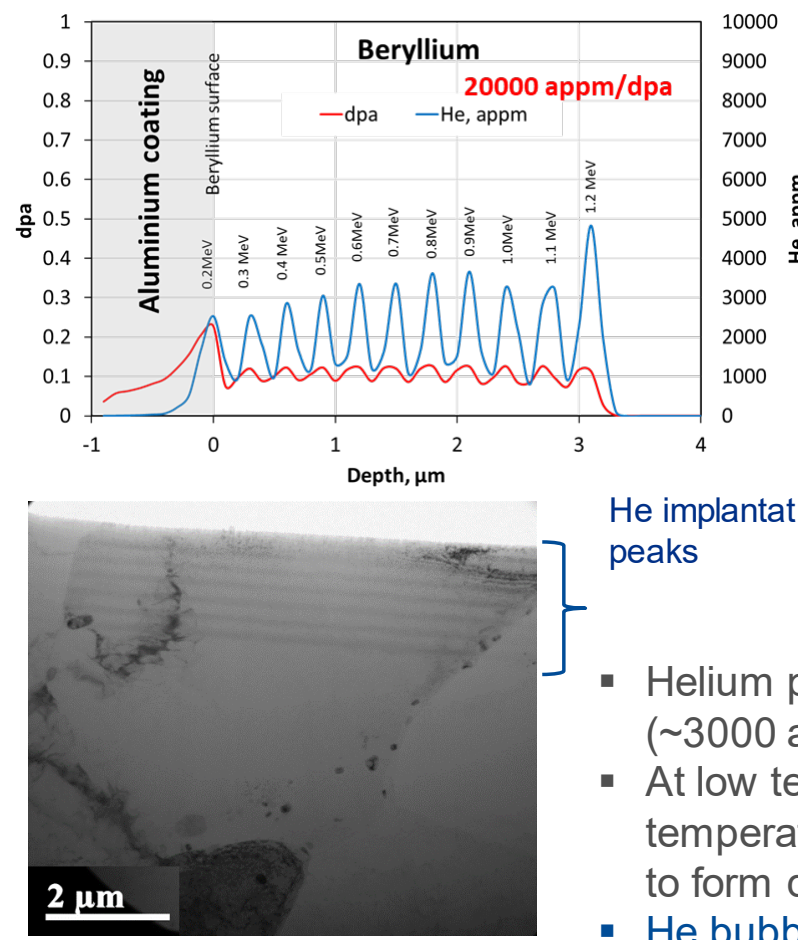

S. Kuksenko, RaDIATE

Collaboration Meeting, 2019

He implantation
$3 \mu \mathrm{m}$ damage layer $\mathrm{T}_{\text {irrad: }}: 50$ and $200{ }^{\circ} \mathrm{C}$ 0.1 DPA, 2000 appm He

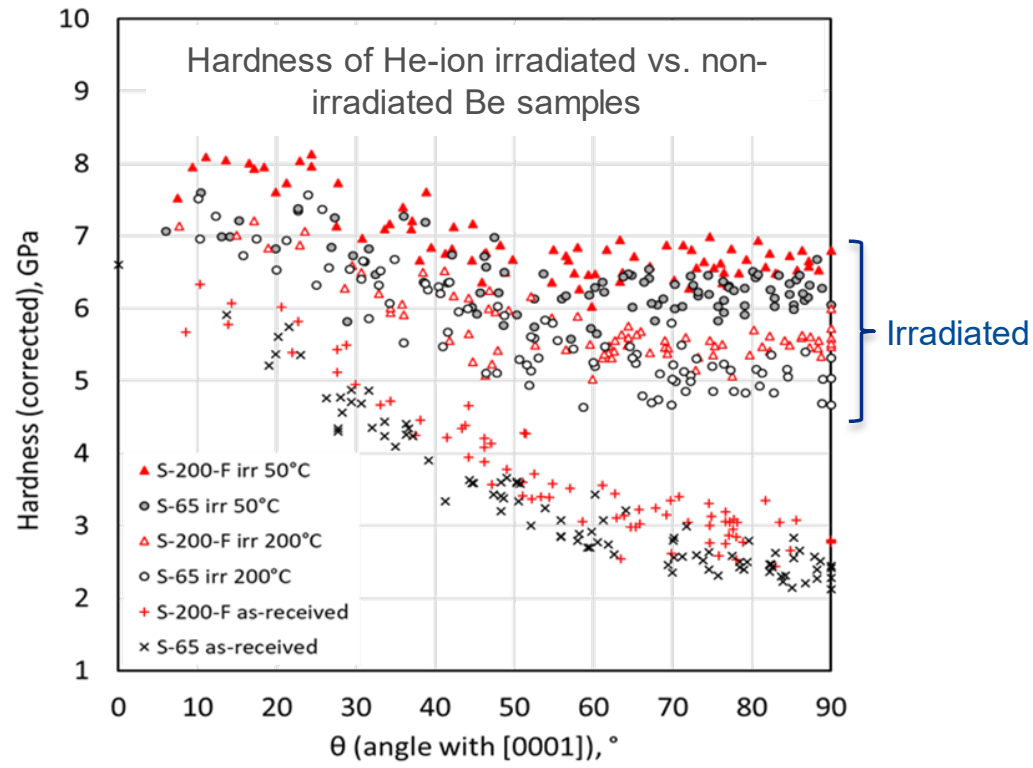

- Helium produced at high rates in Be with high energy proton beams ( 3000 appm/DPA)

- At low temperatures, He atoms do not diffuse while at high temperatures, He atoms become mobile and can fill vacancy clusters to form damaging He bubbles

- He bubbles observed in NuMI Be window after annealing at $360^{\circ} \mathrm{C}$

- However, higher temperatures are generally desired to anneal displacement damage (see hardness plot above)

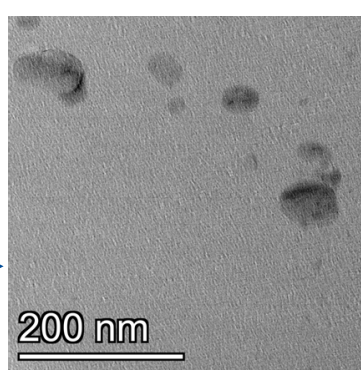




\section{Thermal Shock Experiments at CERN's HiRadMat Facility}

\section{HRMT24 - BeGrid (2015)}

- Observed distinctive thermal shock response for various beryllium grades - 2.8E13 ppp, $\sigma: 0.3 \times 0.28 \mathrm{~mm}$

- Detected plastic strain ratcheting from multiple beam pulses

Successful validation of Be S200FH Johnson-Cook strength model

Profilometry to measure plastic out-of-plane deformations
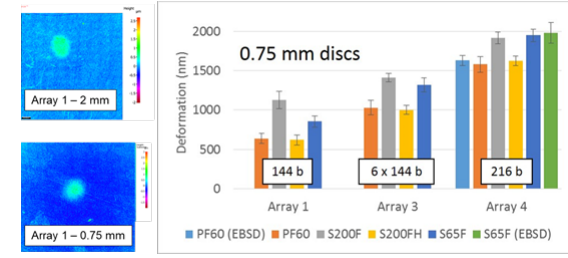

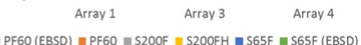

Kuksenko, Oxford

Johnson-Cook model validation

$$
\sigma_{Y}
$$

Confidence in modeling beam-induced

thermomechanical response
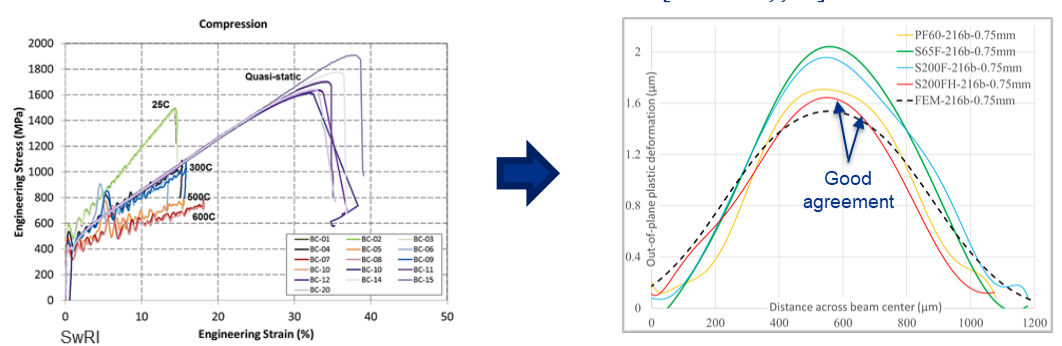

K. Ammigan et al., Phys. Rev. Accel. Beams 22, 044501
HRMT43 - BeGrid2 (2018)

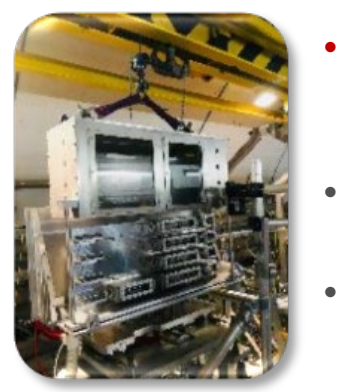

- First and unique test with pre-irradiated material specimens $(\mathrm{Be}, \mathrm{C}, \mathrm{Ti}, \mathrm{Si})$

- $\quad 3.5 \mathrm{E} 13 \mathrm{ppp}, \sigma: 0.27 \times 0.22 \mathrm{~mm}$

- First test on nanofiber electro-spun fiber mats and metal foam ( $\mathrm{SiC}, \mathrm{ZrO}, \mathrm{Al}_{2} \mathrm{O}_{3}$ )

- Dynamic online measurements of graphite cylinders

Online measurements and benchmarking of graphite cylinders
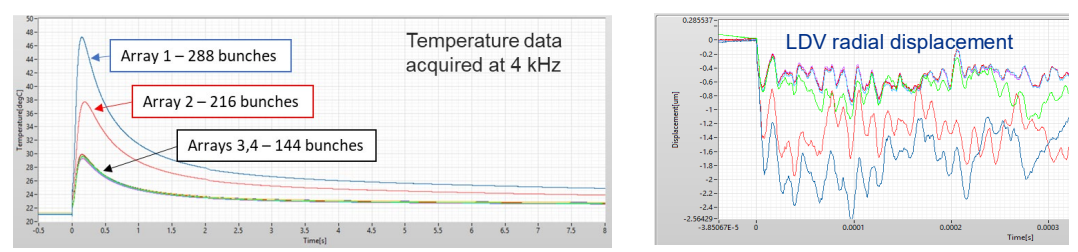

Sigraflex specimens

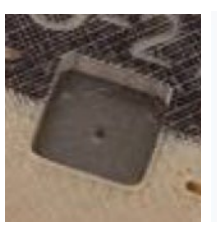

F. Nuiry, CERN

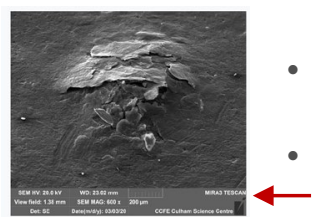

- Numerical simulation benchmarking in progress

- Profilometry and PIE work ongoing at UKAEA MRF, UK

- Damage observed in Sigraflex (LHC dump material) prompted further investigation 


\section{Novel Targetry Materials: Electrospun Nanofibers and HEAs}

Nanofiber electro-spinning at Fermilab

- Nanofiber continuum is discretized at the microscale to allow fibers to absorb and dampen thermal shock, and discontinuity prevents stress wave propagation

- Evidence of radiation damage resistance due to nanopolycrystalline structure of the material
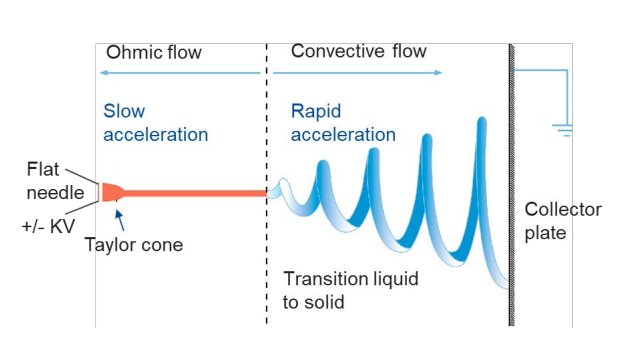

Electrostatically driven

electrospinning process

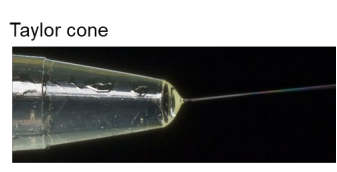

Electro-spinning set-up at Fermilab

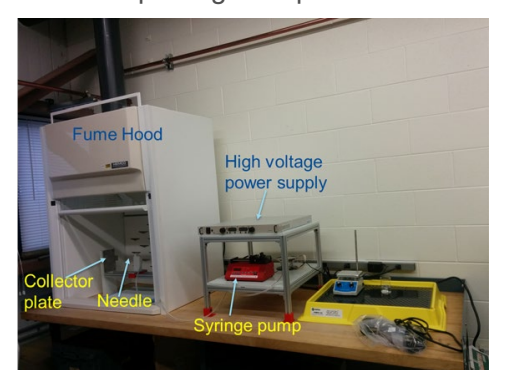

SEM image of Zirconia nanofibers

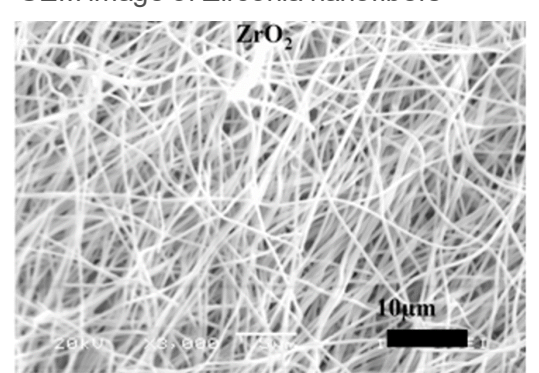

High-Entropy Alloy (HEA) development at UW-M

- Alloys consisting of 3 or more principal elements

- Excellent inherent properties including enhanced radiation damage resistance

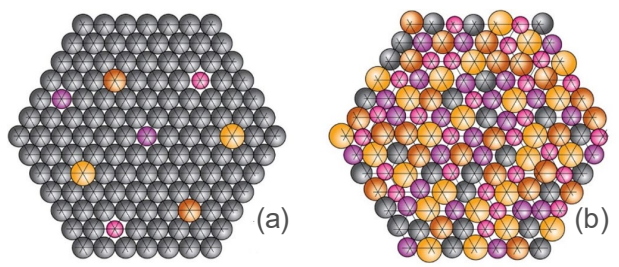

(a) Conventional alloy, (b) High-entropy alloy (Miracle \& Senkov, 2016)
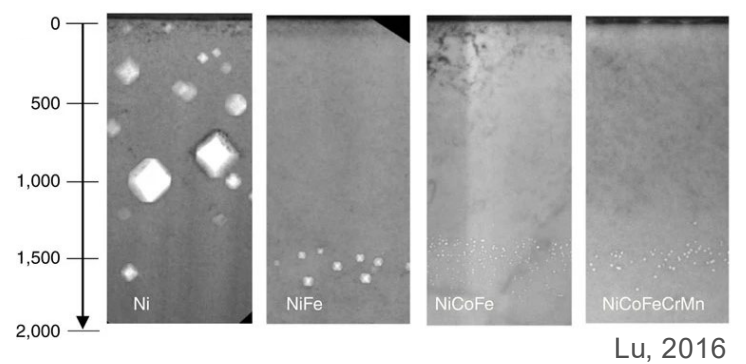

Reduction in irradiationinduced void distribution in nickel and multicomponent HEAs after 3-MeV Ni+ ion irradiation at $773 \mathrm{~K}$ 


\section{Ongoing and Future Target R\&D Activities}

- Complete PIE of BLIP irradiated materials and HiRadMat specimens

- Execute the next HiRadMat experiment in 2022 and ensuing PIE and data analysis

- Ion irradiation studies to correlate with HE-proton (at higher doses and elevated temperatures at MIBL and IRRSUD facility)

- Plan next multi-material HE-proton irradiation within the RaDIATE collaboration framework

- Develop more effective and alternative testing methods to reduce cost and duration of R\&D cycles

- Low-energy ion and electron beam for irradiations, pulsed electron beam for fatigue and thermal shock studies)

- Radiation damage modeling: Ab initio and molecular dynamics material modeling (UW-M, PNNL)

- Develop and qualify novel materials for next-generation target facilities (HEAs, nanofibers, etc.)

- Explore novel targetry concepts: flowing powder, pebble bed, He-cooled spherical array targets 


\section{Summary}

- Future neutrino beams present critical target facility challenges

- Understanding material behavior under intense multi-MW beams is high priority

- Radiation damage effects from lattice disruptions and gas transmutations

- Beam-induced thermal shock limit of materials

- Materials R\&D essential to help design robust targetry components and maximize primary beam power on target and secondary particle production

- Globally coordinated R\&D activities are producing useful results

- Alternative testing methods essential to support R\&D program

- Several novel target concepts and materials are being explored and developed 


\section{Thank you}

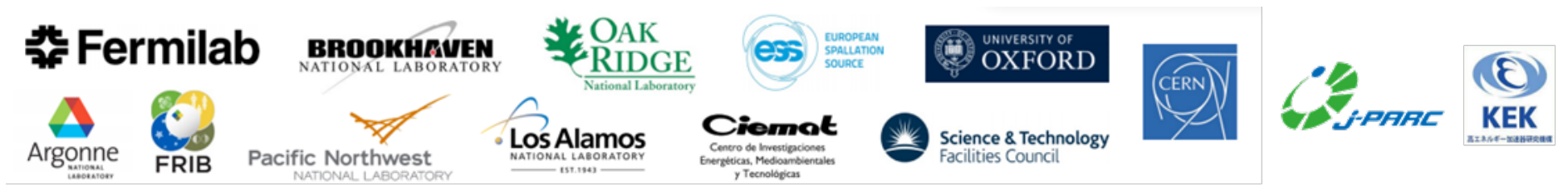

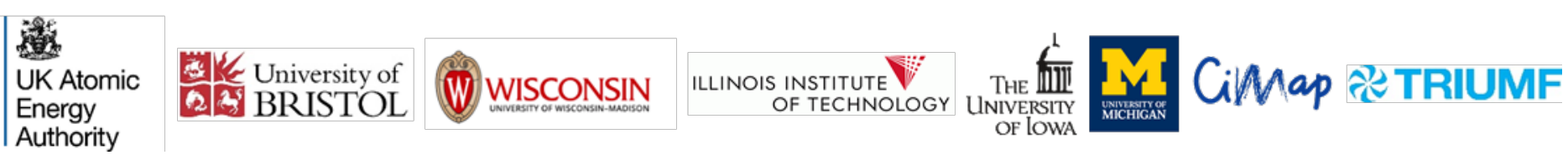

